\title{
Multi-Agent Coordination and Control Testbed for Planning and Scheduling Strategies
}

\author{
Paul Verstraete, Paul Valckenaers, Hendrik Van Brussel, Karuna Hadeli, Bart Saint \\ Germain \\ Department of Mechanical Engineering \\ K.U.Leuven University \\ Leuven, Belgium \\ \{paul.verstraete, paul.valkenaers, hendrik.vanbrussel, hadeli.karuna, \\ bart.saintgermain\}@mech.kuleuven.be
}

\begin{abstract}
The Multi-Agent Coordination and Control (MACC) testbed is a modelling and simulation environment for manufacturing control. It provides benefits to both the research community and industry. It uses a multi-agent manufacturing execution system, developed at PMA.
\end{abstract}

\section{Categories and Subject Descriptors}

I.2.11 [Artificial Intelligence]: Distributed Artificial IntelligenceMultiagent systems

\section{General Terms}

design, experimentation

\section{INTRODUCTION}

The Multi-Agent Coordination and Control (MACC) testbed is a modelling and simulation environment for manufacturing control.

Manufacturing control is the operational level of production planning and control. It is the decision making activity concerned with the short-term and detailed assignment of operations to production resources.

The testbed uses a multi-agent Manufacturing Execution System (MES), developed at K.U.Leuven PMA [1]. A MES is a manufacturing control information system that supports the execution on the shop floor. This support ranges from tracing the execution to supervising the process control systems, the routes that products follow and the assignment of operations to production resources.

Current MES implementations do not support handling of disturbances. Disturbances are handled by operators on the shop floor today. If deliveries are late, clients change orders or machines break down, they try to deal with the problem, respecting the given requirements as good as possible (e.g. due date).

Operators on the shop floor however only have limited insight on how the rest of the system will react as a consequence of their deci-

Permission to make digital or hard copies of all or part of this work for personal or classroom use is granted without fee provided that copies are not made or distributed for profit or commercial advantage and that copies bear this notice and the full citation on the first page. To copy otherwise, to republish, to post on servers or to redistribute to lists, requires prior specific permission and/or a fee.

AAMAS'06 May 8-12 2006, Hakodate, Hokkaido, Japan.

Copyright 2006 ACM 1-59593-303-4/06/0005 ...\$5.00. cisions. Therefore they mainly rely on their experience in dealing with the disturbances they encounter.

Using multi-agent technology, the PMA multi-agent MES supports operators in taking these decisions, improves their insight in the consequences these decisions will have and allows for selforganization.

The testbed consists out of several tools:

- a modelling tool

- a web-based simulation service

- a simulation log browser

Results of the simulation can be twofold: studying the dynamics of the modelled systems or calculating performance criteria.

The testbed tools are written in Java and run on any platform supporting Java.The tools can be started from the website without seperate software installation (http://www.mech.kuleuven.be/benchmarking).

\section{TESTBED ARCHITECTURE}

The architecture of the testbed is structured into three main software components:

\subsection{Test-Bench Assistant}

The Test-Bench Assistant is a visual environment for assisting the designer of a test-bench case in inputting all the main data of the industrial case he/she wants to propose to the scientific and industrial community.

The data, describing an industrial case, comprise [2]:

- The production system taken as a reference (production system model):

- the structural features: the physical configuration of the production system.

* the physical components (workstations, transporters, storage) and their capabilities (sawing, milling, ...).

* the layout of the plant

- the technological features: the process plans of the products being processed in the plant

- optionally, a planning indicating start, end times and the machine to be used for the various operations.

- Dynamic information about the industrial case : 


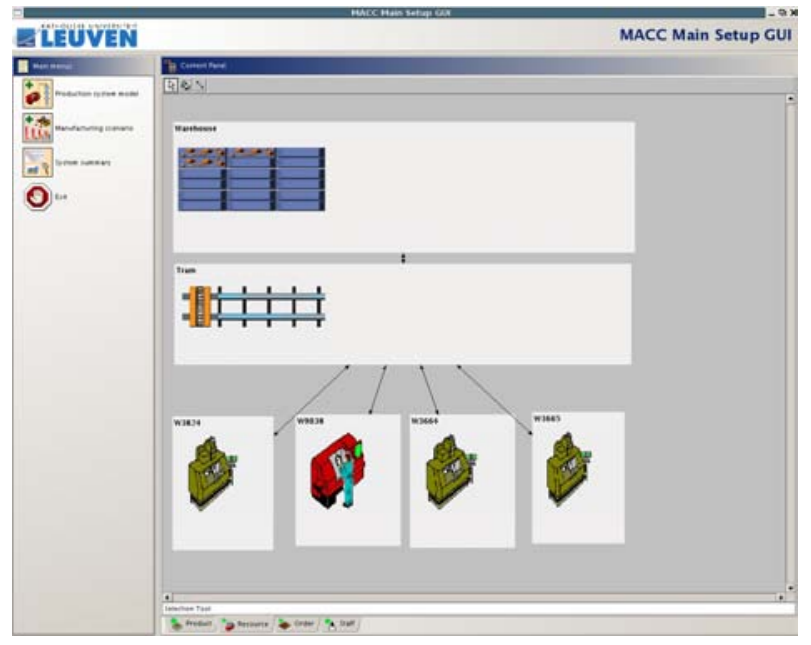

Figure 1: The test bench assistant

- The plant scenario: events or activities related to the functioning of manufacturing physical components (e.g. machine breakdowns, stochastic variations on setup times and operation processing times)

- The operational scenario:

* Production orders

* Expected due dates and release dates

* Disturbances like stock breackage, or changes in orders, due dates or release dates.

\subsection{TestBed Simulator}

A web-based simulation tool for the experimentation, testing and performance analysis of submitted scheduling strategies (without visualisation). The simulation tool takes the input of the test-bench assistant and generates a simulation log, describing the state of the factory and the short term forecasts through the simulation run. Any performance measures can be calculated, based on this log.

\subsection{The log browser}

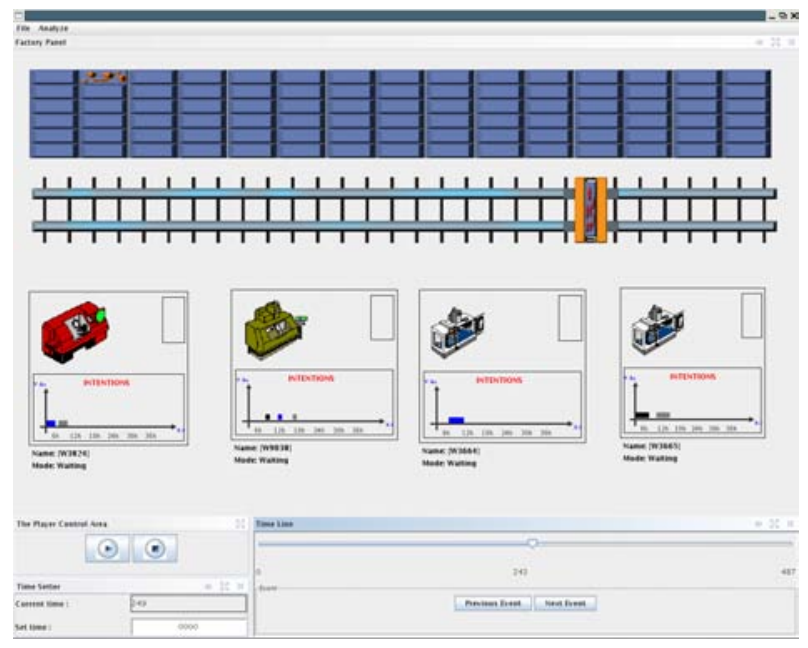

Figure 2: The log browser
The log browser visualizes the simulated factory, the production execution and the short-term forecasts.

A short term forecast indicates the work that has to be carried out in the next shift(s). This forecast automatically adapts in case of changing circumstances (new orders, delays in execution, missing parts, ...).

\section{BENEFITS}

The testbed provides the following benefits:

- Providing a common base for integrating manufacturing control and scheduling strategies into existing systems.

The case model has to be modelled only once and can be combined with different control strategies. If new strategies are available, they can be tested on the case.

The control strategy does not have to solve the whole problem. If no solution is given for part of the problem, the agents in the MES self-organize.

- Provide more insight to operators on the shop floor when taking decisions.

The short term forecast described earlier, supports operators by providing the possible short-term future states of the underlying system. When changing jobs sequences or the starting time of a job (e.g. for maintenance), every entity involved gets an updated forecast.

- Provide more insight to developers in the applicability of their algorithm in various industrial cases.

The testbed allows to collect industrial cases and to make them available to the research community. This shortens the time for conducting experiments significantly and increases the industrial relevance of the results.

\section{FUTURE WORK}

Future efforts aim to expand the possibilities for integrating planning strategies into the MES. For now, the MES follows the planning if it is feasible. If not, it searches a feasible alternative. Subject of further work is to allow the MES to search the feasible alternative that corresponds the most to the original planning [3].

Furthermore, the design of the MES allows to provide plug-ins, e.g. a custom scheduler for a physical component or a custom strategy for chosing between alternatives for a production order. In the future, the test-bed assistant will provide a graphical interface for this task.

This manuscript presents work supported by the K.U.Leuven Research Fund (Concerted Research Action on Agents for Coordination and Control).

\section{REFERENCES}

[1] P Valckenaers and H Van Brussel, "Holonic manufacturing execution systems," CIRP ANNALS-MANUFACTURING TECHNOLOGY, vol. 54, no. 1, pp. 427 - 432, 2005.

[2] S. Cavalieri, L. Bongaerts, M. Taisch, M. Macchi, and J. Wyns, "A benchmark framework for manufacturing control," in Proc. of the Second International Workshop on Intelligent Manufacturing Systems, September 1999.

[3] P. Verstraete and P. Valckenaers, "Cooperating planning and manufacturing execution systems," in INCOM, 2006, submitted. 